\title{
Changes in Dental Hygiene Condition According to the Degree Of Motiviation In A Dental Management Program
}

\author{
Hye Jin $\mathrm{Kim}^{1}$, Hyun Ja Joung ${ }^{2}$ \\ ${ }^{1}$ Professor, Department of Dental Hygiene, Dongeui University in Busan, Kore \\ ${ }^{2}$ Professor, Department of Dental Hygiene Daegu Health College in Daegu, Kore
}

\begin{abstract}
This study was conducted on 150 subjects, who participated in 'the comprehensive clinical dental hygiene management program' for 10 months (Mar-Dec 2015) at the department of dental hygiene of D University. The clinical dental hygiene management consists of dental hygiene assessment, judgment, care plan, and implementation. The study compared the changes in oral hygiene status depending on the motivation of subjects and analyzed the effect of the program on dental hygiene activities. When a subject self-evaluates the dental health status, the degree of motivation increased as the dental health condition evaluation index was lower $(p=0.019)$ and the duration of toothbrushing increased $(p=0.039)$. However, other factors did not have a significant effect on motivation.
\end{abstract}

Keywords : Dental management program, Motivation, Oral health status, Oral care

\section{Introduction}

The introduction of the paper should explain the nature of the problem, previous work, purpose, and the contribution of the paper. The contents of each section may be provided to understand easily about the paper. According to 'the statistics of medical examination expenses evaluation in the first quarter of 2016' announced by Health Insurance Review \& Assessment Service [1], gingivitis and periodontal disease are the third most frequent out-patient diseases. Gingivitis and periodontal disease are inflammatory immune diseases that occur in tissues around the teeth and gradually progress and progress to destroy the alveolar bone. Moreover, they are the second frequent tooth extraction cause [2]. In order to prevent and improve these gingivitis and periodontal diseases, it is required for a patient to actively participate in continuous oral management. In order to enhance oral health, it is necessary to have overall prevention and treatment and change knowledge, attitude, belief, and behavior through education. A motivation can change behavior.

A motivation can be defined as a status to cause a motivation or a status of being motivated. A motivation is classified at extrinsic and intrinsic dimensions. If a behavior occurred due to a reward, it would be called an extrinsic motivator. On the other hand, if a behavior occurred due to an interest, it would be a behavior caused by an intrinsic motivator. Among them, intrinsically motivated behavior is more persistent. 'Intrinsic motivators' associated with special experiences such as confidence, vitality, and self-esteem had a considerable effect on patients' behavior. In order to change the behavior of a patient, learning materials such as educational support media can be utilized to provide learning effects. A patient should be motivated by appropriately using intrinsic and extrinsic motivators with concrete and symbolic attracts. This motivating process is the area of a dental hygienist, who can motivate a patient in the comprehensive clinical dental hygiene management [3]. Comprehensive clinical dental hygiene management [4] consists of dental hygiene assessment, judgment, care plan, and implementation. As a process of dental hygiene implementation, a dental hygienist conducts a comprehensively preventative, therapeutic, and educational work to, ultimately, achieve overall health promotion. One also plays a professional role to support and activate knowledge, attitude, belief, and actions, necessary for improving dental health [5]. As shown, the clinical dental hygiene management is achieved by various methods in practical training and clinic and there are many studies on this topic. However, literature review indicated that the majority of studies focused on comparing and analyzing the changes in oral hygiene. Studies on the importance of an intrinsic motivator were rare.

This study applied the dental hygiene management program of the department of dental hygiene of D University to subjects and compared the dynamics in oral hygiene condition depending on the motivation status. It identified and evaluated the effects of dental hygiene activities. The results will be used as basic information for maintaining the oral health of subjects and active self-management of them.

\subsection{Study Subjects}

\section{Study Methods}

This study was conducted on 150 subjects, who participated in 'the comprehensive clinical dental hygiene management program' for 10 months (Mar-Dec 2015) at the department of dental hygiene of D University. The clinical dental hygiene management [4] consists of dental hygiene assessment, judgment, care 
plan, and implementation. The study compared the changes in oral hygiene status depending on the motivation of subjects and analyzed the effect of the program on dental hygiene activities.

\subsection{Study Methods}

Personal information, medical history, and dental history information was gathered by a survey.

Medical history was classified into hospitalization and operation experience and general disease (전신질환) experience. Dental history was classified into currently experiencing symptoms and scaling experience. Moreover, there were 4 questions related to oral malodor measurement, 7 questions related to oral health-related behaviors and attitude, 14 quality of life questions related to oral health, 20 questions related to dentophobia, 8 questions related to oral hygiene habits, and 8 questions related to drinking and smoking.

Dental management program: Oral health was evaluated by conducting dental exam when all subjects visited the hospital. Test items were skin and mucous membrane at the head and neck, tartar detection, periodontal pocket depth, bleeding test, dental plague (modified O'leary), plaque $\mathrm{pH}$ check, saliva buffering capacity, and oral malodor measurement. The weekly program examined the internal and external oral factor and received feedback through consult and discussion. The program was revised and complemented based on the reevaluation.

\subsection{Analysis Methods}

Collected data was analyzed by using SPSS WIN 18.0 program. Frequency analysis was conducted for general characteristics, dental health awareness, and pre-post comparison. The difference before and after dental hygiene status was analyzed by using the paired t-test. A regression analysis was conducted to analyze the degree of motivation and the factor affecting the motivation.

\subsection{General Characteristics of Subjects}

\section{Results}

Analyses on general characteristics showed that male and female were 43.3 and $56.7 \%$, respectively, age between 21 and 30 was the most frequent (39.3\%), and the majority of subjects were students $(52.0 \%)$, and the highest education level was mostly attending a college $(44.7 \%)$.

$<$ Table $1>$ General Characteristics of the study subjects

\begin{tabular}{|c|c|c|}
\hline Characteristics & Division & $\mathrm{N}(\%)$ \\
\hline Gender & Male & $\begin{array}{l}65(43.3) \\
85(567)\end{array}$ \\
\hline Age & $\begin{array}{c}\leq 20 \\
21-30 \\
31 \leq\end{array}$ & $\begin{array}{l}37(24.7) \\
59(39.3) \\
54(36.0)\end{array}$ \\
\hline Occupation & $\begin{array}{c}\text { Students } \\
\text { Housewives } \\
\text { Office job } \\
\text { Professional job } \\
\text { Else }\end{array}$ & $\begin{array}{c}78(52.0) \\
17(11.3) \\
19(12.7) \\
22(14.7) \\
14(9.3)\end{array}$ \\
\hline Education level & $\begin{array}{c}\leq \text { High school } \\
\text { University } \\
\text { University } \leq\end{array}$ & $\begin{array}{l}50(33.3) \\
67(44.7) \\
33(22.0)\end{array}$ \\
\hline & & $150(100.0)$ \\
\hline
\end{tabular}

\subsection{Changes in Oral Hygiene Status after Conducting the Program}

Oral malodor measurement did not show clear change; from $49.57 \pm 24.35$ (faintly smell odor) to $47.77 \pm 23.74$ (faintly smell odor). Daily toothbrushing frequency increased from $2.57 \pm 0.67$ to $3.00 \pm 0.65$. The duration of toothbrushing (seconds) increased from $137.77 \pm 58.50$ to $152.65 \pm 59.37$. The quantity of toothpaste (g) drew near to $1 \mathrm{~g}$ after the program; from $1.29 \pm 0.54$ to $1.03 \pm 0.22$. Dental hygiene management index increased from $47.83 \pm 20.00$ to $67.36 \pm 17.65$.

$<$ Table 2>After performs oral hygiene program changes

\begin{tabular}{|c|c|c|c|c|}
\hline Division & Before & After & $\mathrm{t}$ & $\mathrm{p}$ \\
\hline Bad breath incentives & $49.57 \pm 24.35$ & $47.77 \pm 23.74$ & 0.805 & 0.422 \\
\hline Days tooth brushing frequency & $2.57 \pm 0.67$ & $3.00 \pm 0.65$ & -7.343 & 0.000 \\
\hline Brushing time & $137.77 \pm 58.50$ & $152.65 \pm 59.37$ & -2.943 & 0.004 \\
\hline Amount of toothpaste & $1.29 \pm 0.54$ & $1.03 \pm 0.22$ & 5.683 & 0.000 \\
\hline Oral hygiene index & $47.83 \pm 20.00$ & $67.36 \pm 17.65$ & - & 0.000 \\
\hline
\end{tabular}




\begin{tabular}{|l|l|l|l|l|}
\hline & & & 15.442 & \\
\hline
\end{tabular}

\subsection{Changes in Oral Malodor according to Motivation}

Oral malodor measurements were $49.57 \pm 24.35$ (before the program) and $47.77 \pm 23.74$ (after the program). There was no significant difference in oral malodor measurement when those before and after the program were compared with the degree of motivation.

<Table 3> Changes in the degree of bad breath incentives

\begin{tabular}{|c|c|c|c|c|c|}
\hline Item & B & Std Error & $\beta$ & T & $\mathrm{p}$ \\
\hline A & -2.326 & 5.688 & -0.039 & -0.409 & 0.683 \\
\hline R & -0.264 & 5.731 & -0.003 & -0.046 & 0.963 \\
\hline C & 0.586 & 6.273 & 0.010 & 0.093 & 0.926 \\
\hline S & -1.186 & 5.442 & -0.023 & -0.218 & 0.828 \\
\hline
\end{tabular}

Item: A(Attention), R(Relevance), C(Confidence), S(Satisfaction)

\subsection{Changes in Dental Hygiene Management Index according to Motivation}

Dental hygiene management index increased from $47.83 \pm 20.00$ (before the program) to $67.36 \pm 17.65$ (after the program). When those before and after the program were compared with the degree of motivation, ' $\mathrm{S}$ ' ( atisfaction) in the ARCS showed a significant correlation with it $(\mathrm{p}=0.023)$.

$<$ Table 4> Oral hygiene index changes according to the degree of motivation

\begin{tabular}{|c|c|c|c|c|c|}
\hline Item & B & Std Error & $\beta$ & $\mathrm{T}$ & $\mathrm{p}$ \\
\hline $\mathrm{A}$ & 1.431 & 3.148 & 0.042 & 0.455 & 0.650 \\
\hline $\mathrm{R}$ & 4.185 & 3.172 & 3.172 & 0.122 & 0.189 \\
\hline $\mathrm{C}$ & 4.242 & 3.472 & 3.472 & 0.124 & 0.224 \\
\hline S & -6.908 & 3.012 & 3.012 & -2.294 & 0.023 \\
\hline
\end{tabular}

Item: A(Attention), R(Relevance), C(Confidence), S(Satisfaction)

\subsection{Changes in the Duration of Toothbrushing according to Motivation}

The duration of toothbrush increased from $137.77 \pm 58.50$ (seconds; before the program) to $152.65 \pm 59.37$ (seconds; after the program). When the result was compared with the degree of motivation, it showed a significant correlation with ' $\mathrm{R}$ (relation)' part in ARCS ( $\mathrm{p}=0.021)$.

$<$ Table 5> Brushing time change according to the degree to incentives

\begin{tabular}{|c|c|c|c|c|c|}
\hline Item & B & Std Error & $\beta$ & $\mathrm{T}$ & $\mathrm{p}$ \\
\hline $\mathrm{A}$ & -8.945 & 12.444 & -0.66 & -0.719 & 0.473 \\
\hline $\mathrm{R}$ & 29.326 & 12.538 & 0.214 & 2.339 & 0.021 \\
\hline $\mathrm{C}$ & 15.175 & 13.723 & 0.111 & 1.106 & 0.271 \\
\hline S & 0.732 & 11.905 & 0.006 & 0.062 & 0.951 \\
\hline
\end{tabular}

Item: $\mathrm{A}($ Attention), $\mathrm{R}$ (Relevance), $\mathrm{C}($ Confidence $), \mathrm{S}$ (Satisfaction)

\subsection{Changes in the quantity of toothpaste according to Motivation}

The quantity of toothpaste ( $\mathrm{g}$ ) was $1.29 \pm 0.54$ (before the program) and $1.03 \pm 0.22$ (after the program) When the result was compared with the degree of motivation, it did not show a significant correlation with ARCS.

$<$ Table 6> It interrupts the amount of change in the degree of motivation

\begin{tabular}{|c|c|c|c|c|c|}
\hline Item & B & Std Error & $\beta$ & $\mathrm{T}$ & $\mathrm{p}$ \\
\hline $\mathrm{A}$ & -0.29 & 0.115 & -0.024 & -0.250 & 0.803 \\
\hline $\mathrm{R}$ & 0.034 & 0.116 & 0.027 & 0.292 & 0.771 \\
\hline $\mathrm{C}$ & -0.025 & 0.127 & -0.020 & -0.198 & 0.844 \\
\hline S & -0.128 & 0.110 & -0.122 & -1.162 & 0.247 \\
\hline
\end{tabular}

Item: $\mathrm{A}($ Attention $), \mathrm{R}($ Relevance $), \mathrm{C}($ Confidence), $\mathrm{S}$ (Satisfaction)

\subsection{Factors Affecting Motivation}

When a subject self-evaluates the dental health status, the degree of motivation increased as the dental health condition evaluation index was lower $(\mathrm{p}=0.019)$ and the duration of toothbrushing increased $(\mathrm{p}=0.039)$. However, other factors did not have a significant effect on motivation.

$<$ Table 7> Factors affecting motivation

\begin{tabular}{|c|c|c|c|c|c|}
\hline Item & B & Std Error & $\beta$ & $\mathrm{t}$ & $\mathrm{p}$ \\
\hline Age & -0.002 & 0.008 & -0.021 & -0.259 & 0.796 \\
\hline
\end{tabular}




\begin{tabular}{|c|c|c|c|c|c|}
\hline Oral Health Status & -0.445 & 0.188 & -0.194 & -2.374 & 0.019 \\
\hline Dental Fear & 0.346 & 0.212 & 0.132 & 1.631 & 0.105 \\
\hline Measurement breath & -0.001 & 0.004 & -0.019 & -0.229 & 0.819 \\
\hline Oral hygiene index & -0.002 & 0.007 & -0.022 & -0.277 & 0.782 \\
\hline Time of tooth brushing & 0.004 & 0.002 & 0.168 & 2.086 & 0.039 \\
\hline The amount of interrupts & -0.253 & 0.207 & -0.099 & -1.224 & 0.223 \\
\hline Oral health behavior & -0.013 & 0.064 & -0.016 & -0.205 & 0.838 \\
\hline
\end{tabular}

\section{Results And Discussion}

Modern dental hygiene activities require various expertise and technology associated with dental disease prevention and dental health promotion. The main job of a dental used to be restricted to dental hygiene education and scaling. However, preventative treatment and dental hygiene management, and factual survey have become a part of duty so dental hygienists are asked to play the role of comprehensive dental hygiene managers. A hygienist enhances the dental health of subjects and meets the needs of them by identifying the dental health problems of dental management program subjects and providing individualized dental hygiene plan with using professional knowledge and methods. Moreover, a hygienist continuously manages dental hygiene based upon evaluation [6]. The dental management program is conducted by various methods in the practice and the clinic. There are many studies related to it [7][8].

However, there were only a few studies evaluating the importance of a subject's intrinsic motivator. This study identified and evaluated the effects of dental hygiene activities by applying dental management program of the department of dental hygiene of D University to subjects and examined the changes in dental hygiene status depending on motivation. Analysis results of general characteristics showed that female was $56.7 \%$, which was more than male, the most frequent age class was between 21 and $30(39.3 \%)$, the student was more than half of occupation $(52.0 \%)$, and attending a college was the highest $(44.7 \%)$. After conducting the dental management program, $45.3 \%$ of respondents said that they 'checked the sugar content when they select a snack', which was an approximately 7.55 times increase compared to before the program. Moreover, $56.7 \%$ of respondents said that they thought that it is 'important' to the question of 'how do you think of choosing snacks containing no sugar?' The intake of snack containing sugar more than once a day decreased to $32.2 \%$. Daily toothbrushing frequency 'more than 3 times per day' increased to $80.7 \%$.

The majority of respondents $(76.0 \%)$ said that they 'replaced toothbrush every three months'. The ratio of using floss increased by 3.25 times; from 21.3 to $69.3 \%$. After conducting a dental management program, oral malodor measurement did not show a clear change. Daily toothbrushing frequency $(3.00 \pm 0.65)$, the duration of toothbrushing (seconds; 152.65 \pm 59.37 ), and dental hygiene management index $(67.36 \pm 17.65)$ increased. The quantity of toothpaste was closer to $1 \mathrm{~g}(1.03 \pm 0.22 \mathrm{~g})$. Comparing with the degree of motivation, the change in oral malodor did not show a significant different; $49.57 \pm 24.35$ (before) and $47.77 \pm 23.74$ (after). However, the dental hygiene management indices were $47.83 \pm 20.00$ (before) and $67.36 \pm 17.65$ (after) and they were significantly correlated with ' $\mathrm{S}$ (satisfaction)' in ARCS ( $\mathrm{p}=0.023)$. The duration of toothbrushing increased from $137.77 \pm 58.50$ (seconds; before) and $152.65 \pm 59.37$ (seconds; after) and it was correlated with ' $\mathrm{R}$ (relation)' in ARCS ( $\mathrm{p}=0.021$ ). The quantity of toothpaste ( $\mathrm{g}$ ) was $1.29 \pm 0.54$ (before) and $1.03 \pm 0.225$ (after) and it did not have a significant correlation with ARCS. It was found that the majority of subjects had incorrect dental hygiene knowledge because they did not receive enough dental hygiene education. To change the condition, high-level dental management program should be provided by learning comprehensive clinical dental activities as well as the education on dental hygiene. The important role of a dental hygienist is to enhance the dental health, meet the requirements, and continue to manage for evaluating, maintaining, and improving the dental hygiene process by confirming the dental health problems of a subject and conduct a designed planned as well as inducing behavioral changed through granting intrinsic motivator [9].

Results revealed that most of the subjects showed positive changes in dental health awareness and dental hygiene condition after receiving the dental management program. When they are compared with the degree of motivation, oral malodor and the quantity of toothpaste did not show significant relationship but dental hygiene indeed and the duration of toothbrushing were significantly correlated with ARCS. It was confirmed that the motivation induction of subjects could influence the dental hygiene condition positively. This study compared the change in dental hygiene condition depending on the degree of motivation of subjects received the in-school practice and emphasized the importance of motivation. The limitation of this study was that it used the subjective motivation criteria and there were only a few items regarding the subjective evaluation of caregiver and the dental hygiene condition according to the degree of motivation. It is believed that various further studies are needed to effectively apply to an oral management program in the future.

\section{References}

[1]. MJ. Cho, ES. Lee, JR. Park, Jy. Jeong et al. Clinical dental hygiene care. 4th, Gomoonsa, 2015, 5-9.

[2]. SY. Lee, HN. Choi. Analysis of case reports based on dental hygiene process. Journal of Korean Society of Dental Hygiene, 11(5), $2011,749-758$. 
[3]. Christoph A. Ramseier, Health Behavior Change in the Dental Practice, Gomoonsa, 2014, 15

[4]. ES. Lee, CH. Lee. A Study on the Oral Hygiene Index Change according to Comprehensive Dental Hygiene Care Treatment. The Korean Journal of Health Service Management, 5(3), 2013, 145-155.

[5]. HY. Oh, CH. Kim, YH. Park, SH. Lim, J. Kim. The comparison on periodontal attitude and oral health promotion behavior by dental hygiene process applies. Journal of Korean Society of Dental Hygiene, 12(5), 2012, 861-870.

[6]. MJ. Jo, ES. Lee, JR. Park. JY. Jeong. Clinical Dental Hygiene Care, Gomoonsa, 2015, 5-9.

[7]. RJ. Park, YK. Lee, HK. Son, MH. Hong. Comparison of oral hygiene status by clinical dental hygiene care performance, Korean Society of Dental Hygiene, 14(1), 2014, 25-32.

[8]. HJ. Noh, MN. Kim, YS. Ahn. Effect of Standardized Oral Health Care Program by Dental Hygiene School Students, Korean Society of Dental Hygiene Science, 12(3), 2012, 287-294.

[9]. YK. Choi, BM. Shin, JH. Son, DY. Park. Effectiveness and Learning Experience of Dental Communication Course Training in Dental Hygiene Schools, Korean Society of Dental Hygiene Science, 16(2), 2016, 134-141. 